\title{
Automated Recognition of Sleep Stages Using Electroencephalograms
}

\author{
Valeriy Bezruk, Egbert Gedat, Katja Holzheimer, Ralf Vandenhouten*
}

\section{Abstract}

The assessment of different sleep stages and their disorders in diseases is an important part of telematic medicine. With an electroencephalogram, the different stages of sleep can be monitored and classified with respect to brain activity. By means of modern data management such as the patient monitor ixTrend, for example, the data can be recorded for long sleep phases and evaluated by a computer using appropriate software, such as Dataplore. Here, a new mathematical model for the automated classification of sleep stages is introduced. The statistical method of autocorrelation, applied to six known sleep stages, was extended by one new class for unknown signals. Due to this new class, it is not necessary to sort all recorded EEG signals into one of the known classes, thereby, minimising the probability of errors. Further, the dependence of the error probability on the duration of the analysed EEG signal was assessed. A minimal error probability of $p_{\min }=0.15$ was detected. Exemplary data for one patient are reported.

\section{Zusammenfassung}

Die Beurteilung der verschiedenen Schlafphasen und deren Störungen bei Erkrankungen spielt eine wichtige Rolle in der telematischen Medizin. Mit einem Elektroenzephalogramm können die Schlafphasen überwacht und in verschiedene Klassen der Hirnaktivität eingeteilt werden. Durch moderne Methoden des Datenmanagements, wie etwa den Patientenmonitor ixTrend, können die Daten über längere Schlafphasen aufgezeichnet und am Computer mit geeigneter Software, etwa Dataplore, analysiert werden. Hier wird ein neues mathematisches Modell zur automatischen Klassifizierung der Schlafphasen vorgestellt. Die statistische Methode der Autokorrelation, angewendet auf sechs bekannte Schlafphasen, wurde erweitert um eine neue Klasse nicht zuordenbarer Muster. Durch diese neue Klasse wird es vermieden, jedes aufgezeichnete EEGMuster in eine der bekannten Klassen hineinzuzwingen. Dadurch wird der Fehler bei der Zuordnung verringert. Weiter wird der Einfluss der Länge des analysierten EEG auf die Fehlerquote der Zuordnung untersucht. Es zeigte sich eine minimale Fehlerquote von $p_{\min }=0,15$. Es werden beispielhafte Daten eines Patienten gezeigt.

\section{INTRODUCTION}

The telematics reasearch group at the TH Wildau has been pursuing a collaboration with ixellence and Philips in the area of telemonitoring for several years. Problems of automated diagnosis support are increasingly emerging. In addition to vital parameters like heart frequency, blood pressure, and ECG the analysis of EEG imposes new demands on signal processing. With an electroencephalogram the sleep stages can be monitored, classified according to brain activity, and screened for disorders due to diseases (Motamedi-Fakhr et al. 2014:21-33). Studying the structure of sleep and its disorders plays an important part in medical telematics. The analysis of the nighttime sleep structure is significant in the diagnosis of brain disorders. In cases of functional diseases of the nervous system and psychoses, sleep disorders are often the main complaint, and sometimes the only one. Examination of the nighttime sleep structures turns out to be also useful in the analysis of the sleep functions and mechanisms. Specifically the sleep structure of patients with ischemic heart diseases is studied in cardiac clinics, since dangerous cardio-vascular system attacks - heart beat disorders, myocardial infarction often develop during the night sleep (Neymark et al. 1985:376).

Changes in the functional condition of the brain entail changes in the nature of its electric activity. The electroencephalogram (EEG) is a good indicator of the brain's level of wakefulness and adequately reflects the depth of sleep. Analysis can also be performed with respect to cardiovascular parameters (Cotuk et al. 2004). Successes achieved in studying sleep organisation are closely related to the use of electroencephalographic examinations. However, recognition of the sleep stages by the EEG records is time consuming, labour intensive, and expensive, since EEG recording of the night sleep takes hundreds of meters of documentation on paper. Moreover, visual analysis involves only descriptive criteria to identify 
the stages of sleep, which lead to the emergence of differing expert views in assessing the transitions between the stages. Sleep structure study objectivation and automation has therefore become a necessity. Various mathematical methods of signal analysis may be applied for this purpose (Bezruk et al. 2005: 45-48, Kravchenko et al. 1991: 138-142, Neymark et al. 1985: 376). Nevertheless, automated determination of the sleep stages by the EEG with the use of mathematical methods is related to certain difficulties, caused by the similarity of the spectral composition of the EEG for separate sleep phases and stages, specifics of correspondent EEG in different people, and the emergence of artifacts.

Different approaches can be applied to solve these problems, characterised by the selected mathematical description (model) of the EEG and the respective recognition algorithm. Spectral methods of the sleep stages recognition can be used, based on an EEG description with expansion factors given by specific base functions (e.g. DEF, Walsh, and Haar) (Neymark et al. 1985:376). Furthermore, the autoregressive model (AR) can be applied to describe the EEG (Zhovna \& Shallom 2008: 2665-2668). It enables the synthesis of autoregressive algorithms for signal recognition (Kravchenko et al. 1991: 138-142). In this research, using the method of known signal recognition is recommended, extended by a class of unknown signals to consider the effects of artifacts (Neymark et al. 1985: 376). This enables reducing the probability of errors while solving the problem of automatic recognition of the sleep stages in real conditions. The suggested autoregressive method of the sleep stages recognition by the EEG is described, as well as some findings of examinations, carried out by statistical simulation with the selected sections for 6 typical stages of sleep from the EEG. A schematic of the method is depicted in Fig. 1.

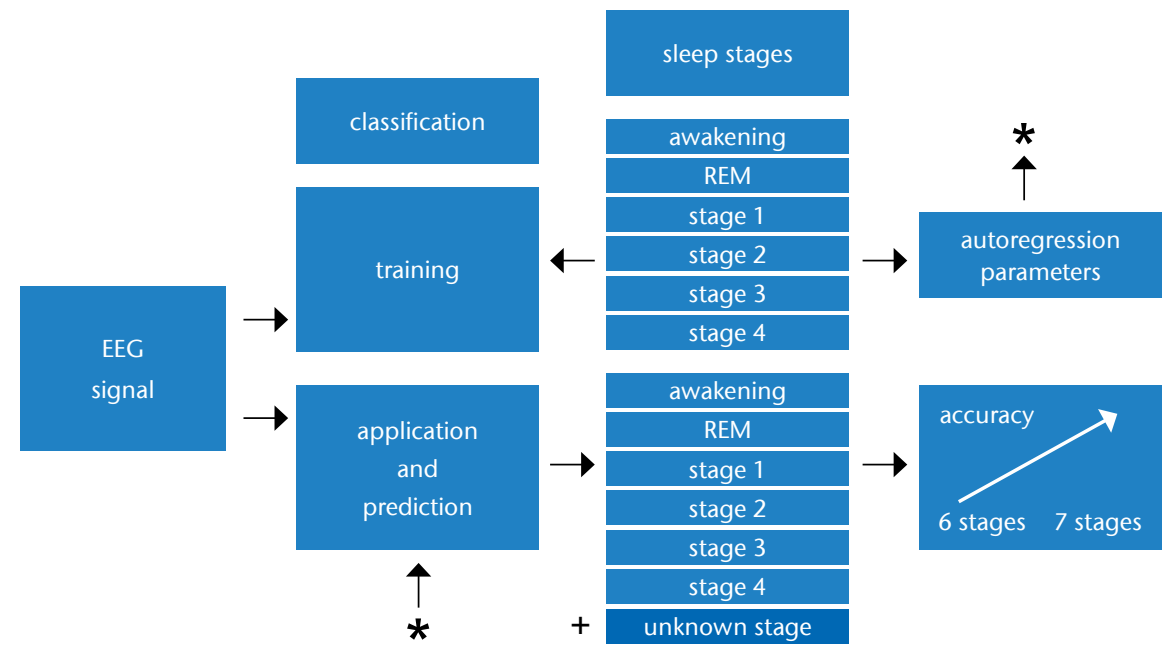

Fig. 1) Schematic of the EEG signal processing. The autoregression algorithm is trained with known sleep stages yielding the autoregression parameters. These are used to analyse unknown EEG signals. The introduction of a seventh class 'unknown stage', increases the accuracy of the method.

\section{EEG MATHEMATICAL MODEL AND THE METHOD OF SLEEP STAGES RECOGNITION}

Analysis of EEG data is mathematically adhered to time-series analysis as in (Vandenhouten et al. 2000: 729-737, Vandenhouten et al. 1999). It is presumed that the EEG is adequately described by the mathematical model as an autoregression process (Kravchenko et al. 1991: 138-142).

(1) $X_{t}=a_{1}^{i} \cdot X_{t-1}+a_{2}^{i} \cdot X_{t-2}+\ldots+a_{p_{i}}^{i} \cdot X_{t-p}+\sigma_{i} v_{t}$

The parameters of the AR model $A_{i}=\left(a_{1}^{i}, a_{2}^{i}, \ldots, a_{p_{i}}^{i}, \sigma_{i}, i, p_{i}\right)$ at any time $t$ meet the stability terms and belong to one of the $M$ classes. It is suggested that the parameters of the AR model, selected to describe the EEG, are known or may be determined based on the classified training of selected values of the EEG sections. It is also thought that the parameters of the EEG model remain stable over significant period of time.

After receiving another reference reading $x_{t}$ of the EEG section under already available reference readings $\tilde{x}_{t}=\left(x_{t}, \ldots, x_{t-T}\right)$ it is necessary to find the class to which the analyzed EEG segment belongs to at $t \geq T$ (where $T$ is the duration of the EEG segment under analysis). In a number of practical cases, the duration of the EEG time interval $T$, which determines the decision, is significantly shorter than the time interval of the AR stability parameters, selected for the EEG description. Then it is possible to suggest a simpler solution for the problem of the automated sleep stage recognition using autoregressive algorithms of the signal recognition. Furthermore, to take the appearance of artifacts appearing in real world conditions into consideration, introduction of an additional $M+1$ - class of unknown signals in the recognition decision procedure is expected. Considering the expression for the multivariate density in Gaussian autoregressive sequences, the algorithm of automated recognition of the given sleep stages of the EEG, together with the now available $M+1$ - class of unknown signals, will look as follows (Kravchenko et al. 1991: 138-142, Bezruk et al. 2005: 45-48): 
(2b) $K_{k}(\vec{x})-K_{i}(\vec{x})+\ln \frac{\left(2 \pi \sigma_{i}\right)^{p_{i}-L}}{\left(2 \pi \sigma_{k}\right)^{p_{k}-L}} \geq \ln \frac{P_{k}}{P_{i}}$

$2 c$

$$
H^{M+1}: K_{k}(\vec{x})>\Lambda_{k}, \quad k=\overline{1, M}
$$

Here, with

$$
2 \mathrm{~d} \quad K_{k}(\vec{x})=\frac{1}{2 \sigma_{k}^{2}} \sum_{l=p=l}^{L}\left[x_{l}-\mu_{k}-\sum_{j=p+1}^{p_{k}} a_{j}^{k}\left(x_{l-j}-\mu_{k}\right)\right]^{2}
$$

a correlation is included in the expression for the multivariate density, determining the standardised forecasting error in the AR model. These $\Lambda_{k}$ are given by

2e $\Lambda_{k}=\ln \frac{(2 \pi)^{\frac{L}{2}} \sigma_{k}^{L-p_{k}} \lambda_{k}}{P_{k}}$

Tab. 1: Autoregression model parameters obtained under the EEG for 6 different sleep stages.

\begin{tabular}{|c|c|c|c|c|c|c|}
\hline & $i=1$ & $i=2$ & $i=3$ & $i=4$ & $i=5$ & $i=6$ \\
\hline$\sigma_{i}$ & 25.4 & 18.3 & 21.5 & 15.7 & 15.9 & 11.2 \\
\hline$a_{1}$ & 0.61 & 0.51 & 0.24 & 0.06 & 0.12 & 0.38 \\
\hline$a_{2}$ & 0.67 & 0.42 & 0.33 & 0.18 & 0.50 & 0.26 \\
\hline$a_{3}$ & 0.75 & 0.37 & 0.25 & 0.13 & 0.11 & 0.33 \\
\hline$a_{4}$ & 0.62 & 0.24 & 0.06 & 0.08 & 0.09 & 0.28 \\
\hline$a_{5}$ & 0.32 & 0.13 & 0.06 & 0.03 & 0.07 & 0.17 \\
\hline$a_{6}$ & 0.16 & 0.17 & 0.17 & 0.04 & 0.04 & 0.12 \\
\hline$a_{7}$ & 0.17 & 0.22 & 0.14 & 0.08 & 0.06 & 0.16 \\
\hline$a_{8}$ & 0.15 & 0.18 & 0.05 & 0.02 & 0.01 & 0.15 \\
\hline$a_{9}$ & 0.09 & 0.13 & 0.05 & 0.04 & 0.01 & 0.09 \\
\hline$a_{10}$ & 0.03 & 0.04 & 0.05 & 0.01 & 0.01 & 0.03 \\
\hline
\end{tabular}

and define threshold values, determined by the provision of the given probabilities of the correct recognition of the given $M$ sleep stages. $p_{k}$ is the order and $a_{j}^{k}$ are the EEG AR model parameters for $k$ sleep stage. According to this, a two step recognition algorithm decision for the $i$ sleep stage applies: provided execution of at least one of the inequalities in (2a), as well as provided the execution of the inequality system in (2b). When inequality $(2 c)$ is executed, the decision for the $M+1$ class of the unknown signals applies. In the recognition algorithm (2), the parameters of the AR models are assumed to be known. Providing they are unknown, they should be determined by the selected classifying training EEG sections.

Thus, this algorithm operates in two modes: training and recognition. In the training mode it is possible to determine the values of the correlation functions, which enable identifying the AR model parameters (in particular, using the Yule Walker equation). In recognition mode, it can be determined to which class (corresponding to the sleep stages) a given EEG with duration $T$ belongs, including the possible option that it belongs to the class of the unknown signals.

Geometrical sense of the rule of decisionmaking according to (2) is that for the EEG of the given sleep stages, separate closed regions are constructed in parameter space. If observations are made in one of these separate regions, the decision is made for the respective sleep stage. Otherwise, the observed EEG section refers to the class of unknown signals.

The decision-making procedure (2) may be extended in case it is necessary to make decisions for EEGs coming from several recording channels. To describe this multi-channel EEG, it is possible to use vector autoregression. Therewith, it is possible to define a recognition algorithm of type equation (2), which allows for the more complicated situation of multi-channel input. The practical specifics and working features of the autoregression signal recognition algorithms using selected Gaussian random signals with different correlation functions were as- 
sessed in (Neymark et al. 1985: 376). From the practical point of view, it is interesting to conduct the study with the suggested algorithm for recognition of given signals under available unknown signals using equation (2) for the problem of recognising stages of sleep by the EEG.

\section{FINDINGS FOR AUTOMATED SLEEP STAGES RECOGNITION BY EEG}

We will provide several results of studying the sleep stage recognition algorithm of equation (2) that were obtained using selected EEGs for the 6 stages of sleep. Table 1 shows the AR model parameters that were found for the EEGs for each of the 6 stages of sleep. The research involved selected EEG sections with the volume of 50,000 reference points for each of the 6 stages of sleep. The parameters were generated according to the classified training decision procedure of equation (2).

Reference sections were used to study practical specifics of solving the problem of automated recognition of the sleep stages by EEG. We also examined the dependency of the quality of recognising sleep stages on the value of the EEG monitoring interval $T$, which determines the decision on the current sleep stage (Fig. 2).

Minimum value of the mean probability of the erroneous recognition of the sleep stages was found $p_{\min }=0.15$. In the result of producing reference EEG selections for the recognition of different stages of sleep the following diagrams of changes in the sleep stages were obtained. They are convenient for the operative analysis of the patient's sleep structure and further diagnostics. One section of the diagram is provided in Fig. 3.

\section{CONCLUSION}

A new method of automated recognition of the given sleep stages by EEG is suggested, introducing the class of unknown signals. The method is based on the EEG description by the mathematical model in the form of autoregression processes. During the process of the EEG recognition, the decision is made either for one of the sleep stages or for

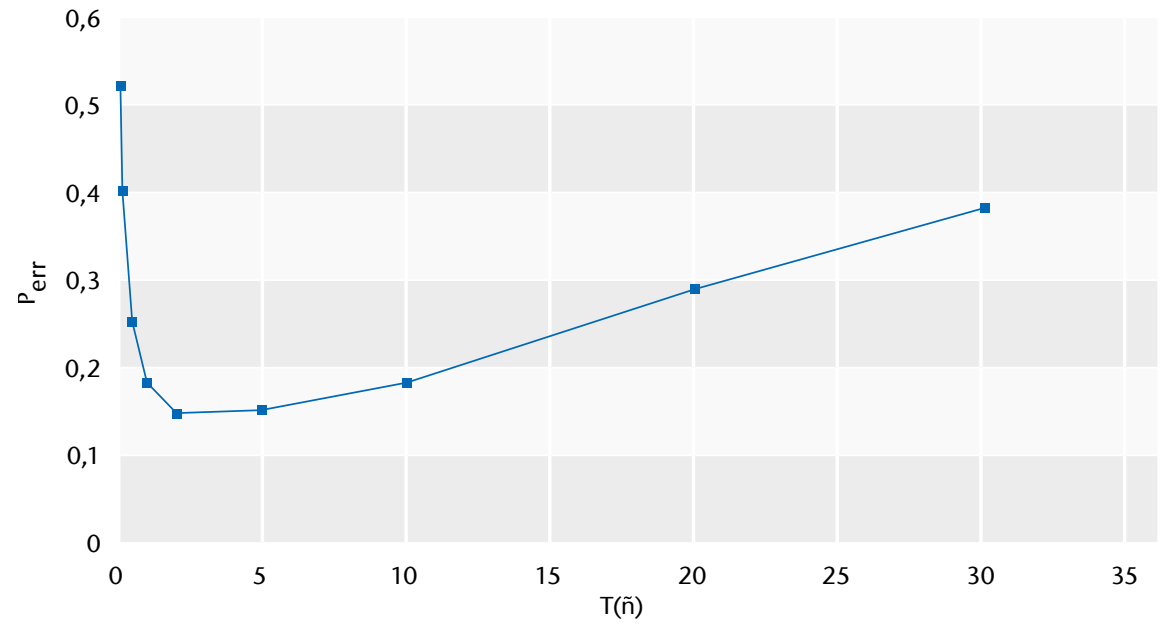

Fig. 2) Dependency of the mean probability of erroneous recognition of the sleep stages on the duration of the EEG interval used to make decisions.

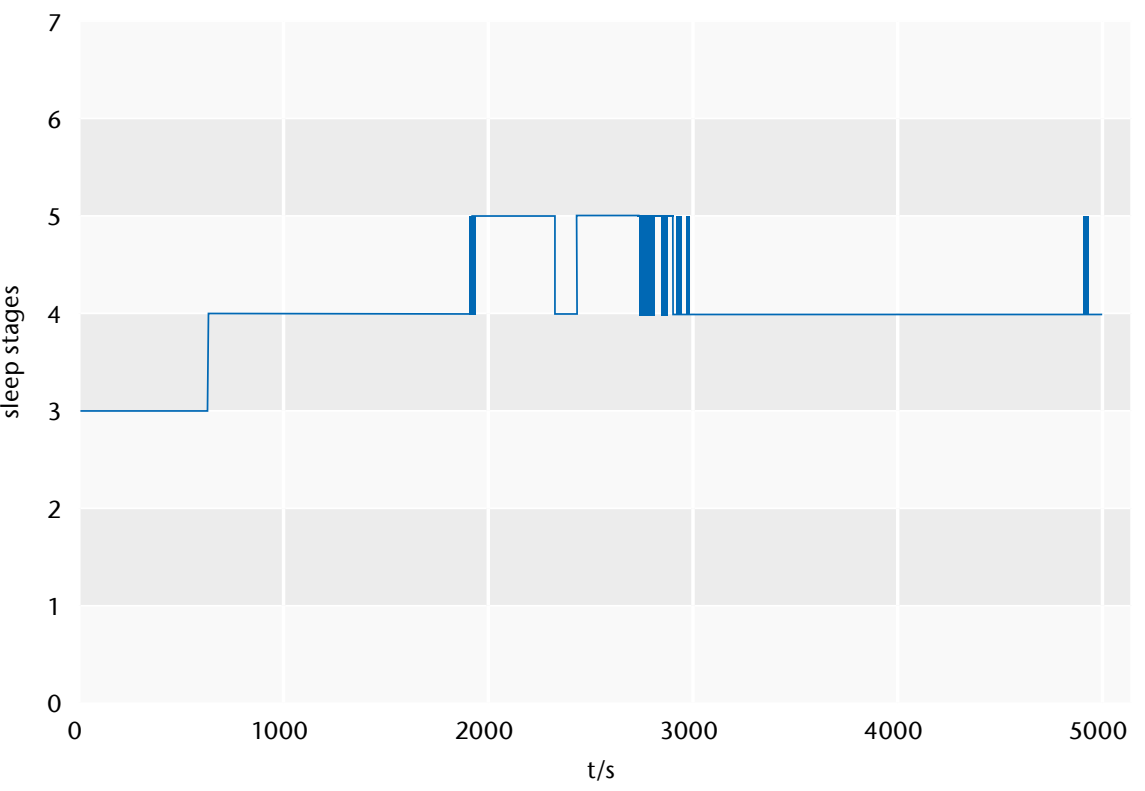

Fig. 3) Diagram of sleep stage $(N=3 \ldots 4 \ldots 5)$ changes on the duration of sleep obtained by the results of the EEG recognition.

the class of unknown observations. This method of recognition enables reduction of the probability of erroneous decisions in real conditions, which infer the emergence of various artifacts. The method of recognising sleep stages by EEG may be used in creating automated systems of information support for diagnosing diseases of the nervous and cardiovascular systems.

The work provides findings of practical specifics of the automated sleep stages recognition by EEG. The study was carried out by statistical simulation using selected EEG sections for 6 typical sleep stages. The dependency of the error in recognising sleep stages on the EEG time interval used for determining the decision was obtained. The research paper also provides time diagrams of changes in the sleep stages, which may be practically useful in the operative evaluation of the night sleep structure. In order to further reduce probability of erroneous recognition of sleep stages, it is advisable to apply EEGs received from several channels (8 or 16) in making decisions. Therewith, it is preferable to use a vector autoregression model for the EEG mathematical description and synthesise the correspondent algorithm of the EEG recognition.

To obtain final recommendations for the practical use of the suggested method of automated sleep stages recognition, it is necessary to conduct 
clinical studies on real night sleep EEGs using signals coming from several channels of the electroencephalograph, as well as taking into account different sources of artifacts, including opening the eyes and changes in the electrode position.

The method is suited for integration in a direct telemonitoring software, ixTrend (Holzheimer 2014b), and the appendant data analysis software, Dataplore (Holzheimer 2014a, Vandenhouten et al. 1999), developed by one of the author's research groups (RV). Here, the EEG signals displayed on standard patient surveillance monitors are tapped and fed into a sophisticated signal analysis environment, providing diverse tools, including statistical and nonlinear analysis.

\section{REFERENCES}

Bezruk VM, Kovalenko NP, Lysenko VA (2005) About one method of the sleep stages recognition based on the autoregression model of electroencephalograms (in Russian). Bionika intellekta Nov.(1):45-48

Cotuk B, Perlitz V, Vandenhouten R, Grebe R, Lambertz M, Petzold ER, Schmid-Schönbein H (2004) Variable $\mathrm{N}: \mathrm{M}$ phase synchronisation between physiological rhythms and the $0.15 \mathrm{~Hz}$ rhythm band during 'relaxed' behavioural states. In: Proceedings of the International Congress on Modeling Mental Processes and Disorders, 24-29 May 2004, Kuşadası, Turkey

Holzheimer K (2014a) Dataplore - Analysis of signals and time series data. https://www.ixellence.com/index. php/en/products/dataplore. Accessed 25 Aug 2014

Holzheimer K (2014b) ixTrend - recording vital signs from Philips IntelliVue patient monitors. https:// www.ixellence.com/index.php/en/products/ixtrend. Accessed 25 Aug 2014

Kravchenko NI, Bezruk VM, Tihonov VA (1991) Recognition of casual signal within the framework of autoregression models. Probabilistic models and processing casual signals and fields (in Russian). UMK VO, Kiev

Motamedi-Fakhr S, Moshrefi-Torbati M, Hill M, Hill CM, White PR (2014) Signal processing techniques applied to human sleep EEG signals - A review. Biomed Signal Process Contr 10:21-33. doi: 10.1016/j.bspc.2013.12.003

Neymark YP, Kogan NJ, Saweljew WP (1985) Pattern recognition in medical diagnosis (in Russian). Nauka, Moscow

Vandenhouten R, Lambertz M, Langhorst P, Grebe R (2000) Nonstationary time-series analysis applied to investigation of brainstem system dynamics. IEEE Tran Biomed Eng 47(6):729-737. doi: 10.1109/10.844220

Vandenhouten R, Rasche M, Parotat E, Lenz A., Fluck M (1999) Dataplore - A Professional Environment for Time Series and Signal Analysis. Datan

Zhovna I, Shallom ID (2008) Automatic detection and classification of sleep stages by multichannel EEG signa modeling. In: IEEE Engineering in Medicine and Biology Society (EMBS) Annual International Conference, 20-25 Aug 2008, Vancouver, BC, pp 2665-2668. doi: 10.1109 / IEMBS.2008.4649750

\section{AUTHORS}

Prof. Dr. Valeriy Bezruk

Head of department Communication networks

Kharkiv National University of Radio Electronics, Ukraine

valeriy_bezruk@ukr.net

Dr. rer. nat. Egbert Gedat

Technische Hochschule Wildau

Forschungsgruppe Telematik

egbert.gedat@th-wildau.de

\section{Katja Holzheimer}

ixellence $\mathrm{GmbH}$

katja.holzheimer@ixellence.com

Prof. Dr. rer. nat. Ralf Vandenhouten

Technische Hochschule Wildau

Forschungsgruppe Telematik

ralf.vandenhouten@th-wildau.de 